\title{
USO DE Trichoderma harzianum Rifai COMO PROMOTOR DE CRECIMIENTO EN PRODUCCIÓN EN VIVERO DE Eucalyptus globulus Labill.
}

\author{
Penon, E.; Craig, E. ${ }^{11}$; Giachino. V.; De Falco, P.; Sobero y Rojo, M.; \\ Rodriguez, P. y Tornatore, A.
}

\section{RESUMEN}

En Argentina se producen anualmente un promedio de 55 millones de plantines forestales de distintas especies de pinos y eucaliptos principalmente. La gran heterogeneidad de crecimiento del material resultante y la alta incidencia de enfermedades en la etapa de vivero (producidas por hongos como Rhizoctonia spp., Pythium spp., Fusarium spp. y Botrytis cinerea), así como la mortandad o menor crecimiento derivado de condiciones de estrés hídrico durante las primeras etapas de la implantación a campo son problemas conocidos en el sector forestal. En la producción se utilizan grandes cantidades de agroquímicos y nuevas tecnologías asociadas con sustratos inertes y fertiirrigación. El mercado forestal a nivel mundial exige plantas de calidad, pero cuya producción sea sustentable teniendo en cuenta aspectos ambientales, económicos y sociales.

El objetivo de este estudio fue evaluar durante el ciclo de cultivo en vivero de Eucalyptus globulus Labill, el uso del hongo Trichoderma harzianum Rifai como una alternativa productiva de bajo impacto ambiental en su acción como promotor de crecimiento. Se utilizó sustrato de siembra forestal compuesto principalmente por turba y perlas volcánicas (Terrafertil) esterilizado previamente. Se mezcló el sustrato de siembra con Trichoderma harzianum y se aplicó fertilizante de liberación lenta a todos los tratamientos ( $8 \mathrm{~g} / \mathrm{bandeja}$ ). Las formulaciones del hongo fueron en talco y en turba y en las dosis recomendadas por los marbetes. Se sembraron semillas de Eucalyptus globulus de rodal semillero, de procedencia local (INTA 25 de Mayo, Provincia de Buenos Aires). Se utilizaron bandejas de 40 celdas de $90 \mathrm{ml}$ y el diseño fue completamente aleatorizado con 6 repeticiones: T1 Testigo, T2 Trichoderma harzianum en talco 10g/bandeja (10 x $10^{8}$ unidades colonia / bandeja), T3 Trichoderma harzianum en talco $20 \mathrm{~g} / \mathrm{bandeja}\left(20 \times 10^{8}\right.$ unidades colonia/ bandeja), T4 Trichoderma harzianum cepa Th1 en turba al $5 \%$ en volumen (16 $\mathrm{x}$ $10^{9}$ unidades colonia/ bandeja), T5 Trichoderma harzianum cepa Th1 en turba al $10 \%$ en volumen (32 $\times 10^{9}$ unidades colonia/ bandeja). Para evaluar crecimiento y desarrollo de las plantas, a los 5 meses, se midieron: altura, diámetro al cuello, Índice de Dickson, biomasa aérea y biomasa radical.

Al principio y al final del ensayo se determinó el número de unidades formadoras de colonia de Trichoderma harzianum. Los datos fueron analizados estadísticamente por ANOVA y LSD. En todos los casos se utilizó el programa estadístico INFOSTAT, 2009 ®. En el análisis de varianza se observó que todas las variables son significativas excepto la biomasa radical. Las variables que mejor respuesta tuvieron a los tratamientos son la altura y la biomasa aérea. Al final del ensayo, los tratamientos T4 (cepa Th1 con 4,26×107 ufc/g) y T3 (cepa T22 a 2,66×106 ufc/g) mostraron plantas significativamente más altas que el testigo $(+15 \%)$ y de mayor biomasa aérea $(+$ $40 \%)$, respecto al testigo $(p<0.05)$. Respecto a la evolución de las unidades formadoras del hongo durante el ensayo, T3, T4 y T5 tuvieron alta sobrevivencia, llegando al final del ensayo con concentraciones, aunque menores que las iniciales, consideradas efectivas. Las cepas Th1 y T22 de Trichoderma harzianum promovieron el crecimiento de la altura y biomasa aérea de plantas en vivero de Eucalyptus globulus. Es importante evaluar el tipo de formulación del hongo y su sobrevivencia en las distintas condiciones de cultivo de plantas en vivero para poder recomendar diferentes alternativas de manejo.

Palabras clave: Eucalyptus globulus, Viveros, Trichoderma harzianum, promotor crecimiento.

\footnotetext{
${ }^{11}$ Universidad Nacional de Luján, Argentina. craigelena@yahoo.com.ar
} 


\section{SUMMARY}

Argentina produces an annual average of 55 million different species forest seedlings, mainly pine and eucalypts species. The vast heterogeneity of resulting material growth and the high incidence of disease in the nursery stage (produced by fungi such as Rhizoctonia spp., Pythium spp., Fusarium spp., and Botrytis cinerea) as well as the mortality or lower growth derived of water stress conditions during the early plantation stages are well known issues in the forest sector. For producing plants large amounts of chemicals and new technologies associated with inert substrates and fertiirrigation are used. The global forest markets demands quality plants, but produced under sustainable methods considering environmental, economic and social matters.

The study objectives were to evaluate the nursery culture cycle in Eucalyptus globulus Labill, the use of Trichoderma harzianum Rifai fungi strains as a low environmental impact productive alternative and their action as a growth promoter. Tree planting substrate composed primarily of peat and volcanic pearls (Terrafertil) previously sterilized was used. Substrate was mixed with Trichoderma harzianum and slow release fertilizer was applied to all treatments $(8 \mathrm{~g} /$ tray). Fungi formulations were talc and peat and the doses recommended by the tags. Seeds were from local source (INTA May 25, Province of Buenos Aires) seed orchard stands. Used trays were 40 cells of $90 \mathrm{ml}$ each under a completely randomized design with 6 replications: T1 Control, T2 Trichoderma harzianum in talc $10 \mathrm{~g} /$ tray ( $10 \times 10^{8}$ colony units / tray), T3 Trichoderma harzianum in talc $20 \mathrm{~g} /$ tray $\left(20 \times 10^{8}\right.$ colony units/tray), T4 Trichoderma harzianum strain in peat Th1 $5 \%$ by volume (16 x $10^{9}$ colony units/tray), T 5 Trichoderma harzianum strain Th1 in peat $10 \%$ by volume $\left(32 \times 10^{9}\right.$ units colony tray). To evaluate plants growth and development, at 5 months, height, collar diameter, Dickson Index, aboveground biomass and root biomass were measured.

At the beginning and the end of the assay the number of colony forming units of Trichoderma harzianum was determined. The data were statistically analyzed by ANOVA and LSD. In all cases the statistical program INFOSTAT, $2009 \AA$ was used. In the variance analysis was observed that all variables are significant except root biomass. Variables that had better response to treatment are height and biomass. At the end of the trial, under the treatments T4 (Th1 strain with $4.26 \times 107 \mathrm{cfu} / \mathrm{g})$ and T3 (T22 strain to $2.66 \times 106 \mathrm{cfu} / \mathrm{g})$ plants were significantly higher than the control $(+15 \%)$ and also higher in biomass $(+40 \%)$, compared to the control $(p<0.05)$. Regarding to the the fungi evolution in forming units during the test, T3, T4 and T5 had high survival, reaching the end of the test concentrations, although lower than the initial considered effective. Th1 and T22 Trichoderma harzianum strains promoted the growth in height and plant biomass in the nursery seedlings. It is important to evaluate the fungi formulation type and its survival in the different nursery plants culture conditions to recommend different management alternatives.

Key Words: Eucalyptus globulus, nursery, Trichoderma harzianum, growth promotion. 


\section{INTRODUCCIÓN}

En Argentina se producen anualmente un promedio de 55 millones de plantines forestales de distintas especies de pinos y eucaliptos, principalmente. Existe una gran heterogeneidad de calidad del material producido y una alta incidencia de enfermedades producidas por hongos en la etapa de vivero.

El mercado forestal a nivel nacional y mundial exige plantas de calidad, pero cuya producción sea sustentable teniendo en cuenta aspectos ambientales, económicos y sociales. Los microorganismos con efectos benéficos sobre las plantas tienen un potencial considerable como biofertilizantes y como agentes de biocontrol.

Pueden distinguirse tres grandes grupos: fijadores de nitrógeno, hongos micorrízicos y promotores del crecimiento vegetal, entre los que se encuentran bacterias y hongos (Kloeper et al., 1989).

La gran mayoría de los microorganismos del suelo tienen la función de descomponer la materia orgánica del suelo, pero algunos son capaces de eliminar microorganismos patógenos de raíces. Este tipo de patógenos puede no causar síntomas evidentes de enfermedad pero sí disminuir el crecimiento y rendimiento de las plantas, por lo tanto, unos de los mecanismos que tienen los hongos para promover el crecimiento de las plantas es precisamente eliminando los patógenos que lo deprimen. Este no es el único mecanismo de los hongos promotores del crecimiento, también son capaces de solubilizar micronutrientes, de incrementar la absorción y de producir reguladores de crecimiento vegetal. Tienen la capacidad de bloquear sitios de infección de los patógenos, ya que colonizan en forma competitiva las raíces. También puede haber una misma especie de hongo que se comporta tanto como promotor del crecimiento como antagonista.

El género Trichoderma fue introducido por Persoon alrededor de 200 años atrás y se trata de un hongo anamórfico aislado principalmente del suelo como descomponedor de materia orgánica. La actividad micoparasítica y la producción de antibióticos, fueron demostradas por primera vez en Trichoderma por Weinding en 1932 y algunas de las modernas aplicaciones biotecnológicas de este hongo como agente de biocontrol son derivadas de estos primeros trabajos.

Recientemente, algunos autores han podido identificar modos de acción de Trichoderma spp. como biocontrolador e identificaron mecanismos explicados por acción de enrollamientoestrangulación de las hifas de Trichoderma sobre las hifas del patógeno Rhizoctonia solani y la secreción por parte del biocontrolador de inhibidores hidrosolubles que detienen el desarrollo del patógeno (Anees et al., 2010).

Para el caso específico de los hongos promotores del crecimiento del género Trichoderma, su aplicación ha producido en varias especies incremento de la velocidad y porcentaje de germinación, reducción de los efectos causados por condiciones de estrés ambiental (Björkman et al., 1998), aumento de la altura de las plantas, aumento de peso seco y tamaño de las raíces, adelantamiento de los momentos de floración y cosecha, aumento del número de flores por planta y mayor tamaño de los frutos (Kleifeld y Chet, 1992; Varaschin et al., 2000; Gasoni et al., 2001). Sin embargo, solo algunos de los mecanismos involucrados están dilucidados.

\section{OBJETIVO}

El objetivo de este estudio fue evaluar durante el ciclo de cultivo en vivero de Eucalyptus globulus Labill el uso del hongo Trichoderma harzianum Rifai en su acción como promotor de crecimiento, como una alternativa productiva de bajo impacto ambiental que minimice el uso de agroquímicos. 


\section{MATERIAL Y MÉTODO}

La producción de plantas y su evaluación se realizó en el invernáculo del sector forestal de la Universidad Nacional de Lujan, ubicada en Luján, a $34^{\circ} 35^{\prime}$ LS y $59^{\circ}$ LO, Buenos Aires, Argentina.

Para la crianza de las plantas, se utilizaron bandejas de 40 celdas de $90 \mathrm{ml}$, se utilizó sustrato forestal comercial esterilizado al que se agregó fertilizante de liberación lenta para todos los tratamientos.

Se sembraron semillas de Eucalyptus globulus de rodal semillero, de procedencia local (INTA 25 de Mayo, Provincia de Buenos Aires, Argentina).

El diseño fue completamente aleatorizado con 6 repeticiones. En los tratamientos de Trichoderma se utilizaron las cepas Th1 y T22. Las formulaciones del hongo fueron en talco y en turba en distintas dosis (Cuadro $N^{\circ} 1$ ), con los siguientes tratamientos:

\section{T1: Testigo}

T2: Trichoderma harzianum cepa T22 en talco $10 \mathrm{~g} / \mathrm{bandeja}\left(10 \times 10^{8}\right.$ unidades colonia / bandeja)

T3: Trichoderma harzianum cepa T22 en talco $20 \mathrm{~g} / \mathrm{bandeja}\left(20 \times 10^{8}\right.$ unidades colonia/ bandeja)

T4: Trichoderma harzianum cepa Th1 en turba al 5\% en volumen $\left(16 \times 10^{9}\right.$ unidades colonia/ bandeja)

T5: Trichoderma harzianum cepa Th1 en turba al 10\% en volumen (32 x $10^{9}$ unidades colonia/ bandeja)

No se realizaron aplicaciones de plaguicidas durante el desarrollo del ensayo. Para evaluar el crecimiento y desarrollo de las plantas, a los 5 meses se midieron: altura, biomasa aérea y biomasa radical.

Al principio y al final del ensayo se determinó el número de unidades formadoras de colonia de Trichoderma harzianum.

Los datos fueron analizados estadísticamente por ANOVA y test de medias LSD. En todos los casos se utilizó el programa estadístico INFOSTAT, 2009 ®.

\section{TRATAMIENTOS APLICADOS EN EL ENSAYO}

\begin{tabular}{|l|l|}
\hline Tratamientos & \multicolumn{1}{c|}{ Dosis de Trichoderma Inicial } \\
\hline T1 - Testigo & Cero \\
\hline T2 - $10 \mathrm{~g}$ talco/bandeja & $10 \times 10^{8}$ unidades colonia / bandeja - Cepa T22 \\
\hline T3 - $20 \mathrm{~g}$ talco/bandeja & $20 \times 10^{8}$ unidades colonia/ bandeja - Cepa T22 \\
\hline T4 - 5\% turba en volumen & $16 \times 10^{9}$ unidades colonia/ bandeja - Cepa Th1 \\
\hline T5 $-10 \%$ turba en volumen & $32 \times 10^{9}$ unidades colonia/ bandeja - Cepa Th1 \\
\hline
\end{tabular}




\section{RESULTADOS}

En el análisis de varianza se observó que las variables que mejor respuesta mostraron a los tratamientos fueron la altura, el diámetro del cuello, la biomasa aérea y el índice de Dickson.

En la primera evaluación de altura y biomasa total de las plantas, a los 3 meses de la siembra, se observa que los tratamientos T4 y T5 formulados con turba, presentan plantas significativamente más altas que el testigo $(+24 \%)$ y mayor biomasa aérea $(+37 \%)$ respecto del testigo $(p<0.001)$ (Cuadro $\left.N^{\circ} 2\right)$.

En la evaluación final en tanto, a los 5 meses de edad de las plantas, los tratamientos T3 , T4 y T5 mostraron plantas significativamente más altas que el testigo (+15\%), de mayor diámetro del cuello $(+33 \%)$ y de mayor biomasa aérea $(+40 \%)$, respecto al testigo $(\mathrm{p}<0.05)$ Cuadro $\mathrm{N}^{\circ} 2$ y Figura $\mathrm{N}^{\circ} 1$ ).

En la biomasa radicular no se observó diferencia significativa entre tratamientos.

Cuadro $\mathrm{N}^{\circ} 2$

ALTURA DE LAS PLANTAS A LOS 3 Y 5 MESES

\begin{tabular}{|c|r|c|c|c|}
\hline \multirow{2}{*}{ Tratamientos } & \multicolumn{2}{|c|}{3 meses } & \multicolumn{2}{c|}{5 meses } \\
\cline { 2 - 5 } & $\begin{array}{c}\text { Medias } \\
\text { (cm) }\end{array}$ & Test LSD & $\begin{array}{c}\text { Medias } \\
\text { (cm) }\end{array}$ & Test LSD \\
\hline T2 & 15,00 & A & 23,18 & A \\
\hline T1 & 15,58 & A & 25,57 & AB \\
\hline T3 & 17,13 & B & 28,30 & BC \\
\hline T5 & 19,33 & C & 29,45 & C \\
\hline T4 & 19,53 & C & 29,48 & C \\
\hline
\end{tabular}

LSD: Distinta letra indica diferencia significativa ( $p: 0,05)$

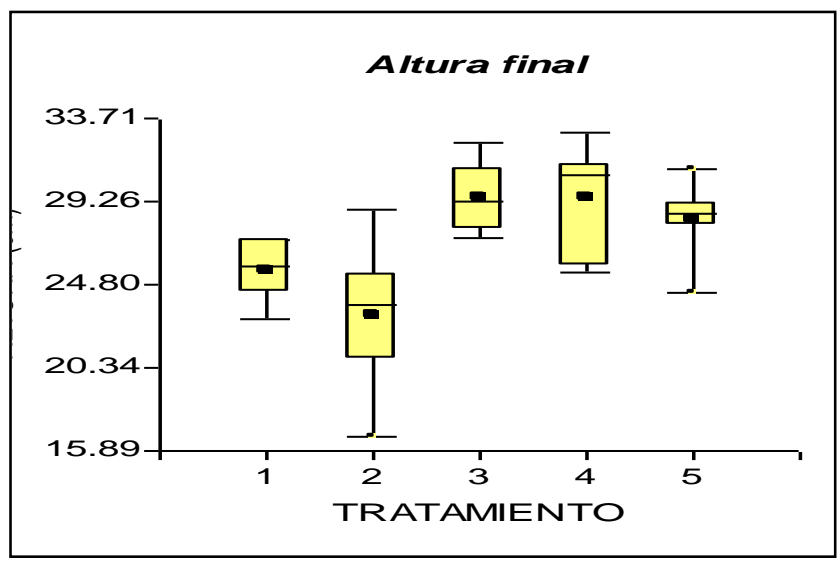

Las cajas indican los cuartiles 25 y 75 . Los puntos centrales la media.

Figura $\mathbf{N}^{\circ} 1$

ALTURA DE LAS PLANTAS A LOS 5 MESES 


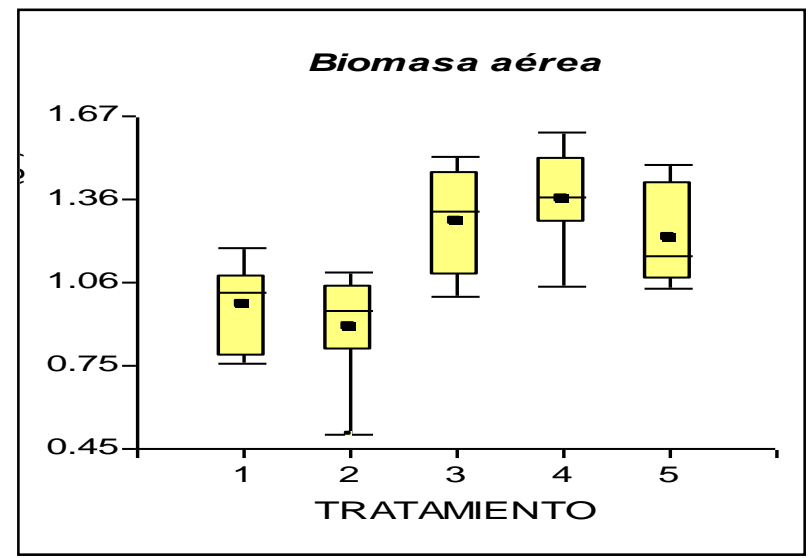

Las cajas indican los cuartiles 25 y 75.

Los puntos centrales la media.

Figura $\mathrm{N}^{\circ} 2$

BIOMASA TOTAL DE LAS PLANTAS A LOS 5 MESES

Para el índice de Dickson solo el tratamiento 4 se diferencia testigo (T1). Los tratamientos 3, 4 y 5 alcanzan valores superiores al testigo incrementando su calidad (Olivo y Buduba, 2006) mejorando potencialmente la sobrevida de la planta en implantación (Cuadro $N^{\circ} 3$ y Figura $\mathrm{N}^{\circ} 3$ ).

En el Cuadro $\mathrm{N}^{\circ} 4$ se muestran los valores de concentración final de $T$. harzianum. La evolución de las unidades formadoras del hongo durante el ensayo indica que T4 y T5 tuvieron buena sobrevivencia, partiendo de $10^{9}$ unidades colonia/ bandeja de la Cepa Th1 llegaron al final del ensayo con concentraciones de $10^{5}$, las que aunque menores que las iniciales pueden considerarse efectivas.

Es de destacar que la cepa T22 tuvo una disminución de unidades de formadoras de colonias más marcada, con una caída desde $10^{8}$ hasta llegar a $10^{3}$.

\section{CUADRO $\mathrm{N}^{\circ} 3$}

ÍNDICE DE DICKSON DE LAS PLANTAS A LOS 5 MESES

\begin{tabular}{|c|r|l|}
\hline Tratamiento & Medias & LSD \\
\hline T2 & 0,10 & $\mathrm{~A}$ \\
\hline T1 & 0,11 & $\mathrm{AB}$ \\
\hline T3 & 0,12 & $\mathrm{ABC}$ \\
\hline T5 & 0,13 & $\mathrm{BC}$ \\
\hline T4 & 0,14 & $\mathrm{C}$ \\
\hline
\end{tabular}

LSD: Distinta letra indica diferencia significativa ( $p 0,05)$ 


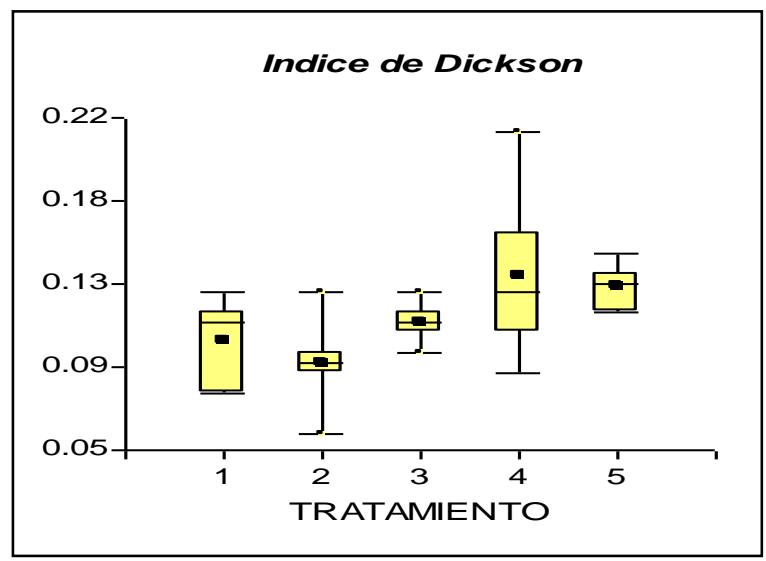

Las cajas indican los cuartiles 25 y 75.

Los puntos centrales la media

Figura $N^{\circ} 3$

INDICE DE DICKSON DE LAS PLANTAS A LOS 5 MESES

Cuadro $\mathrm{N}^{\circ} 4$
CONCENTRACIÓN FINAL DE Trichoderma harzianum

\begin{tabular}{|c|l|}
\hline Tratamiento & \multicolumn{1}{|c|}{ Concentración Final } \\
\hline T1 & Cero \\
\hline T2 & $1 \times 10^{3}$ unidades colonia/ bandeja \\
\hline T3 & $1 \times 10^{3}$ unidades colonia/ bandeja \\
\hline T4 & $1 \times 10^{5}$ unidades colonia/ bandeja \\
\hline T5 & $4 \times 10^{5}$ unidades colonia/ bandeja \\
\hline
\end{tabular}

\section{CONCLUSIONES}

Las cepas Th1 y T22 (mayor dosis) de Trichoderma harzianum promovieron el crecimiento en altura, diámetro del cuello y biomasa aérea de plantas en vivero de Eucalyptus globulus.

El efecto fue más notorio a los 3 meses desde la siembra.

Los mayores índices de Dickson en el tratamiento 4 definen una planta de mejor calidad y una posible mayor supervivencia a campo. La mayor heterogeneidad de índice en el tratamiento 4 puede deberse a la dificultad de lograr una distribución homogénea del formulado en turba en el sustrato.

Es importante evaluar el tipo de formulación del hongo y su sobrevivencia en las distintas 
condiciones de cultivo de plantas para poder recomendar distintas posibilidades de manejo del mismo en el vivero.

La sobrevivencia de Trichoderma harzianum resultó ser mayor en la formulación sobre turba.

\section{REFERENCIAS}

Anees, Muhammad; Tronsmo, Arne; Edel-Hermann, Veronique; Gordon Hjeljord, Linda; Heraud, Cecile and Steinberg, Christian, 2010. Characterization of Field Isolates of Trichoderma Antagonistic Against Rhizoctonia solani. Fungal Biology: 114 (2010) Pags. 691 - 701.

Bjorkman, T.; Blanchard, L. M. and Harman, G. E., 1998. Growth Enhancement of Shrunken-2 (Sh2) Sweet Corn by Trichoderma harzianum 1295-22: Effect of Environmental Stress. J. Amer. Soc. Hort. Sci. 123(1): 35-40.

Gasoni, L.; Cozzi, J.; Kobayashi, K.; Yossen, V.; Zumelzu, G.; Babbit, S.; and Kahn, N., 2001. Yield Response of Lectucce and Potato to Bacterial and Fungal Inoculants Under Field Conditions in Cordoba (Argentina). Journal in Plant Disease and Protection. 108, 530 - 535.

Kleifeld, O. and Chet, I., 1992. Trichoderma harzianum - Interaction with Plants and Effect on Growth Response. Plant and Soil 144: 267-272.

Kloepper, J. W.; Lifshitz, R. and Zablotowicz, R. M., 1989. Free Living Bacterial Inocula for Enhancing Crop Productívity. Tibtech 7: 39-44 (1989).

Olivo, Verónica y Buduba, Carlos, 2006. Influencia de Seis Sustratos en el Crecimiento de Pinus ponderosa Producido en Contenedores Bajo Condiciones de Invernáculo. Bosque 27(3): 267-271, 2006.

Varaschin, C.; Astiz Gassó, M. y Properi, A., 2000. Growth Promotion with Trichoderma spp. Formulations in Four Crops During Early Stages. Abstracts $5^{\text {th }}$ International Pgpr Workshop, 137. Córdoba, Argentina.

Yossen, V.; Zumelzu, G.; Gasoni, L. y Cozzi, J., 1999. Eficiencia de La Solarización y Agentes Promotores de Crecimiento de las Plantas en el Rendimiento de Acelga (Beta vulgaris L.) - XXIV Congreso de la Sociedad Mexicana de Fitopatología y X Congreso Latinoamericano de Fitopatología. México. Abs. 114. 